\title{
Intra-operative use of biological products - Are we aware of their derivatives?
}

\author{
Navdeep Bhamra ${ }^{1}$, Karan Jolly ${ }^{1}$, Adnan Darr ${ }^{1}$, Duncan Bowyer ${ }^{2}$, and Shahzada Ahmed ${ }^{3}$ \\ ${ }^{1}$ Royal Wolverhampton Hospitals NHS Trust \\ ${ }^{2}$ Shrewsbury and Telford Hospital NHS Trust \\ ${ }^{3}$ University Hospitals Birmingham NHS Foundation Trust
}

June 16, 2021

\begin{abstract}
Introduction: Global medical advances within healthcare have subsequently led to the widespread introduction of biological products such as grafts, haemostats, and sealants. Although these products have been used for many decades, this subject is frequently not discussed during the consent process and remains an area of contention. Methods: A nationwide confidential online survey was distributed to UK-based junior registrars (ST3-5), senior registrars (ST6-8), post-CCT fellows, specialist associates/staff grade doctors and consultants working in general/vascular surgery, neurosurgery, otolaryngology, oral \& maxillofacial surgery and plastic surgery. Results: Data was collected from a total of 308 survey respondents. Biological derivatives were correctly identified in surgical products by only $25 \%$ of survey respondents, only $19 \%$ stated that they regularly consent for use of these products. Our results demonstrate that most participants in this study do not routinely consent (81\%) to the intra-operative use of biological materials. An overwhelming $74 \%$ of participants agreed that further education on the intraoperative use of biological materials would be valuable. Discussion: This study highlights deficiencies in knowledge that results in potential ethical compromise of the consenting process for surgical procedures. A solution to this would be for clinicians to increase their awareness via educational platforms and to incorporate an additional statement on the consent form which addresses the potential intraoperative use of biological products and what their derivatives may be. Conclusion: Modernising the current consent process to reflect the development and use of surgical biological products will help to ensure improved patient satisfaction, fewer future legal implications as well as a better surgeon-patient relationship.
\end{abstract}

Intra-operative use of biological products - are we aware of their derivatives?

Dr Navdeep Bhamra - MBChB, Department of Otolaryngology, The Royal Wolverhampton NHS Trust, UK. - study conception and design, analysis and interpretation, data collection, writing and critical revision of article.

navdeepbhamra@gmail.com

Mr. Karan Jolly - MBChB, FRCS (ORL-HNS), Department of Otolaryngology, The Royal Wolverhampton NHS Trust, UK. - study conception and design, analysis and interpretation, data collection, writing of article.

kjolly@nhs.net

Mr. Adnan Darr - BSc (Hons), MSc, MBChB, FRCS (ORL-HNS), Department of Otolaryngology, The Royal Wolverhampton NHS Trust, UK.- study conception and design, analysis and interpretation, data collection, critical revision of article.

adnan.darr@nhs.net 
Mr. Duncan J Bowyer - MBChB, FRCS (ORL-HNS), Department of Otolaryngology, Shrewsbury and Telford Hospital NHS Trust, UK.- study conception and critical revision of article.

duncan.bowyer@sath.nhs.uk

Mr. Shahzada K Ahmed - BSc (Hons) DLO MBChB, FRCS (ORL-HNS), PhD, Department of Otolaryngology, University Hospitals Birmingham NHS Trust, UK. - study conception and critical revision of article.

shahz.ahmed@nhs.net

All authors have read and approved the paper.

Corresponding author:

Dr Navdeep Bhamra - The Royal Wolverhampton NHS Trust, UK, WV10 0QP - navdeepbhamra@gmail.com.

Dr Navdeep Bhamra assumes full responsibility for the integrity of the content of this paper.

No conflicts of interest or funding to be declared.

No ethical approval was required for this study.

\section{Abstract}

INTRODUCTION Global medical advances within healthcare have subsequently led to the widespread introduction of biological products such as grafts, haemostats, and sealants. Although these products have been used for many decades, this subject is frequently not discussed during the consent process and remains an area of contention.

\section{METHODS}

A nationwide confidential online survey was distributed to UK-based junior registrars (ST3-5), senior registrars (ST6-8), post-CCT fellows, specialist associates/staff grade doctors and consultants working in general/vascular surgery, neurosurgery, otolaryngology, oral \& maxillofacial surgery and plastic surgery.

\section{RESULTS}

Data was collected from a total of 308 survey respondents. Biological derivatives were correctly identified in surgical products by only $25 \%$ of survey respondents, only $19 \%$ stated that they regularly consent for use of these products. Our results demonstrate that most participants in this study do not routinely consent (81\%) to the intra-operative use of biological materials. An overwhelming $74 \%$ of participants agreed that further education on the intra-operative use of biological materials would be valuable.

\section{DISCUSSION}

This study highlights deficiencies in knowledge that results in potential compromise of the consenting process for surgical procedures. A solution to this would be for clinicians to increase their awareness via educational platforms and to incorporate an additional statement on the consent form which addresses the potential intraoperative use of biological products and what their derivatives may be.

\section{CONCLUSION}

Modernising the current consent process to reflect the development and use of surgical biological products will help to ensure improved patient satisfaction, fewer future legal implications as well as a better surgeonpatient relationship.

MeSH Keywords: Biological products, consent, education, surgery.

What is known about this topic: 
Global medical advances within healthcare have subsequently led to the widespread introduction of biological products such as grafts, haemostats, and sealants. Although these products have been used for many decades, this subject is frequently not discussed during the consent process and remains an area of contention.

A recent study demonstrated that in 534 patient respondents, $44 \%$ wanted to be informed if biological products were to be used and $17 \%$ objected to their use.

\section{What does this article add?}

As demonstrated by our observational study, this may partly be due to a lack of awareness amongst surgeons of the contents of these products, highlighting a need for increased education.

The authors recommend that the use of biological products should be added to a surgical consent, allowing surgeons to have an open and transparent discussion, whilst upholding their duty to ensure that their clinical practice is patient centred.

INTRODUCTION: Global medical advances within the field of healthcare have subsequently led to the widespread introduction of biological products. Medication, dressings, implants and tissue grafts derived from human or animal material have become part of routine practice.11Jolly, K., Darr, A., Aslanidou, A., Bowyer, D., \& Ahmed, S. (2019). The intra-operative use of biological products: A multi-centre regional patient perspective of a potential consenting conundrum. Clinical Otolaryngology. doi:10.1111/coa.13367 Although these products have been used by doctors for many decades, this subject is frequently not discussed during the consent process and remains a controversial topic.22Enoch, S., Shaaban, H., Dunn, KW. (2005). Informed consent should be obtained from patients to use products (skin substitutes) and dressings containing biological material. Journal of Medical Ethics, 31(1), 2-6. doi:10.1136/jme.2003.005272 '33Eriksson, A., Burcharth, J. \& Rosenberg, J. Animal derived products may conflict with religious patients' beliefs. BMC Med Ethics 14, 48 (2013). https://doi.org/10.1186/1472-6939-14-48 The majority of biological materials used intra-operatively in modern surgery contain porcine or bovine derivatives, the most common of which is gelatin. ${ }^{1}$ Gelatin is found in collagen extracted from dermal and skeletal structures of cows (bovine) and pigs (porcine). The use of gelatin has been evaluated by different faith groups, however general consensus remains a contentious issue. ${ }^{3}$

Broadly speaking biological materials can be divided into three main categories based upon their primary use: grafts, haemostats, and sealants (Table 1). The evolution of new surgical techniques has been facilitated by the development of a range of materials used as grafts and sealants to repair defects and promote wound healing, as well as haemostats to stop bleeding and reduce infection. Consideration is required with their use to ensure that it does not conflict with the patient's ethical, personal, or religious beliefs. It has been documented that Buddhists, Christians (including of Jehovah's witnesses) and Jews accept the use of dressings, implants or medication which are of animal or human origin. ${ }^{3}$ Amongst other faiths, Hindus and Sikhs do not permit the use of any products derived from bovine material ${ }^{3,} 44$ Sattar, S. P., Ahmed, M. S., Majeed, F., \& Petty, F. (2004). Inert Medication Ingredients Causing Nonadherence Due to Religious Beliefs. Annals of Pharmacotherapy, 38(4), 621-624. doi:10.1345/aph.1d324, and Muslims object to the use of porcine-derived materials. However, in general, most faiths agree that in an emergency life-threatening situation the use of any product biological or otherwise is permitted.55Easterbrook, C. (2008). Porcine and Bovine Surgical Products. Archives of Surgery, 143(4), 366.doi:10.1001/archsurg.143.4.366 The major exception to this is in the Jehovah's witness community where it is strictly forbidden to receive primary human blood components (red blood cells, plasma, platelets) ${ }^{1}$, however the use of human-derived mesh is unclear.66Dixon JL, Smalley MG. Jehova's Witnesses: the surgical/ethical challenge. JAMA 1981;246:24712472. A recent study demonstrated that in 534 patient respondents, $44 \%$ wanted to be informed if biological products were to be used and $17 \%$ objected to their use. The reason for objection was multifactorial and often not motivated by religious beliefs. ${ }^{1}$

Owing to the routine use of biological products in surgical practice and patient's stated desire to be informed if this is to be the case, it is essential that this forms part of the consent process. Anecdotally the authors found that the use of these products are not routinely being discussed pre-operatively with patients in the 
United Kingdom (UK); we believe that may partially be due to a lack of knowledge of the constituents of biological materials by the clinician.

We sought to investigate on a national scale what proportion of surgeons from a range of specialties routinely consent for the intra-operative use of biological materials, whilst assessing awareness of these products and their derivatives. We hope this study will strengthen and support our belief that informed consent for the use of biological products intra-operatively should be added to the standard consent form template for all surgery.

\section{METHOD:}

A nationwide confidential online survey (Table 2) was distributed to UK-based junior registrars (ST3-5), senior registrars (ST6-8), post-CCT fellows, specialist associates/staff grade doctors and consultants working in general/vascular surgery, neurosurgery, otolaryngology, oral \& maxillofacial surgery and plastic surgery. Dissemination platforms included social media and local and national trust based internal mail. Demographic data including the participant's grade, region of employment and parent speciality were collected, all respondents remaining anonymous. The survey was tailored to each speciality, with specific questions about materials that reflected commonly used products in their surgical practice. Each participant was given the name of three biological materials which would be used in their speciality and asked to correctly identify the derivative. These questions equally represented the three categories of biological material we have previously outlined (grafts, haemostats, and sealants). Each participant was also asked whether they currently routinely consent for the use of biological material intra-operatively and if they felt that further education in this area would be of value for clinicians.

The survey was created online and distributed via a weblink using social media and email correspondence. Data was analysed using Microsoft Excel. This weblink survey was live for 4 months from November 2019 to February 2020.

RESULTS: Data was collected from a total of 308 survey respondents.

Of the respondents, $34 \%$ were consultants $(n=105), 29 \%$ senior registrars and post-CCT fellows $(n=88)$, $25 \%$ junior registrars $(n=77)$ and $12 \%$ specialist associates/staff grades $(n=38)$. The largest proportion of responses were obtained within the West Midlands deanery $(n=71,23 \%)$ and then by London deaneries $(\mathrm{n}=46,15 \%)$. The breakdown for the responses collected in other deaneries are summarised below (Table $3)$.

General and vascular surgery was the most represented speciality $(\mathrm{n}=120,39 \%)$, followed by otolaryngology $(\mathrm{n}=109,35 \%)$, neurosurgery $(\mathrm{n}=34,11 \%)$, plastic surgery $(\mathrm{n}=24,8 \%)$ and lastly oral \& maxillofacial surgery $(\mathrm{n}=21,7 \%)$, (Table 4).

Biological derivatives were correctly identified in surgical products by only $25 \%$ of survey respondents. Within this, 80 survey respondents identified the correct biological derivative for grafts (26\%), 62 for haemostats (20\%) and 85 for sealants (28\%). The correct response rate for all the materials by speciality is summarised in table 4. Our results demonstrate a poor awareness about the constituents of the products surgeons commonly use across all specialties.

There was no observed significance in correct response rate between the grade of surgeon. Overall, all grades demonstrated poor knowledge in this area (Table 4).

Of the total 308 survey participants, 19\% stated that they regularly consent for use of these products. Analysing the individual surgical specialties included in this survey, otolaryngologists most commonly consent routinely for the use of biological materials during surgery $(23 \%)$, with oral and maxillofacial surgeons most infrequently (10\%). An overwhelming $74 \%$ of participants agreed that further education on the intraoperative use of biological materials would be valuable (Table 5).

\section{DISCUSSION:}


The use of biological products in medicine has become routine but remains controversial and the subject of debate. ${ }^{5}$ Patients wish to know the derivative of any biological products that may be used, which itself raises the issue of informed consent prior to their use. ${ }^{1}$ Due to a patient's ethical, religious or personal beliefs the use of these biological products without consent is morally flawed. Clinicians tend to underestimate the impact and importance of religion in their patients' lives and the implications of this upon their care.11Sulmasy DP. The healer's calling: a spirituality for physicians and other health care professionals. New York: Paulist Press; 1997 Ignoring the impact of religion on a patient's decision is likely to lead to a breakdown in the trust between clinician and patient.22Silvestri GA, Knittig S, Zoller JS, Nietert PJ. Importance of faith on medical decisions regarding cancer care. J Clin Oncol 2003; 21:1379-1382.

Whilst religion remains a major influence on the objection of the medicinal use of biological products, there has been an increase in people who self-identify as vegan and also object to the use of animal derived products. In a 2018 Ipsos Mori study commissioned by The Vegan Society, the number of vegans in the United Kingdom had increased from $0.25 \%$ of the population to $1.16 \%$ in 2018 , which equates to approximately 600,000 adults.33The Vegan Society. Food and You Surveyhttps://www.vegansociety.com/my-account/thevegan/issue-3-2018/survey (Accessed 01/04/2020) This number may have increased further given the recent publicity and awareness-raising campaigns promoting veganism and vegetarianism. Of the biological products we have included within this survey, only one product (Surgicel) is plant-based, with the remainder including a human or animal derivative. This can restrict treatment options in those who specifically object to the use of products of human or animal origin, potentially altering surgical outcomes. Alongside this, we must not overlook that objection to the use of biological products need not be driven by religious or dietary motives but more a matter of personal ethical and moral principles.

This study has demonstrated that there is a lack of awareness amongst surgeons of biological products and their derivatives, implying that patients are also often unaware and thus may not be consented for their use. This lack of awareness can be appropriately addressed by educating clinicians, which can be delivered efficiently through educational platforms such as e-learning modules. This notion is supported by $74 \%$ of our cohort who agreed that further education was required in this subject area (Table 5).

The design of this study may have given rise to a degree of participant bias, as respondents may have been more likely to complete the survey if they had sufficient knowledge within the field. A greater geographic spread, as well as numbers in specialties other than general surgery/ vascular surgery and otolaryngology may have highlighted specific deficits in knowledge.

Our results demonstrate that most participants in this study do not routinely consent (81\%) to the intraoperative use of biological materials (Table 5). The authors hope that this study highlights deficiencies in knowledge that results in potential compromise of the consenting process for surgical procedures. A simple solution to this would be for clinicians to increase their awareness in this area and to incorporate an additional statement on the consent form which addresses the potential intraoperative use of biological products and what their derivatives may be.

CONCLUSION: Although the intra-operative use of biological materials has become routine, the consent process does not reflect this. Patients wish to know which products may be used during their procedure; however, this is not being routinely discussed pre-operatively. This may partly be due to a lack of awareness amongst surgeons of the contents of these products, highlighting a need for increased education. The authors recommend that the use of biological products should be added to a surgical consent, allowing surgeons to have an open and transparent discussion, whilst upholding their duty to ensure that their clinical practice is patient centred. Modernising the current consent process to reflect the development and use of surgical biological products will help to ensure improved patient satisfaction, fewer future legal implications as well as a better surgeon-patient relationship.

No further acknowledgements.

\section{Hosted file}


BM Figure 1.docx available at https://authorea.com/users/323901/articles/526477-intraoperative-use-of-biological-products-are-we-aware-of-their-derivatives

\section{Hosted file}

BM Figure 2.docx available at https://authorea.com/users/323901/articles/526477-intraoperative-use-of-biological-products-are-we-aware-of-their-derivatives

\section{Hosted file}

BM Figure 3.docx available at https://authorea.com/users/323901/articles/526477-intraoperative-use-of-biological-products-are-we-aware-of-their-derivatives

\section{Hosted file}

BM Figure 4a.docx available at https://authorea.com/users/323901/articles/526477-intraoperative-use-of-biological-products-are-we-aware-of-their-derivatives

\section{Hosted file}

BM Figure 5a.docx available at https://authorea.com/users/323901/articles/526477-intraoperative-use-of-biological-products-are-we-aware-of-their-derivatives 